\title{
Immigrant Arrival and Tuberculosis among Large Immigrant- and Refugee-Receiving Countries, 2005-2009
}

\author{
Zachary White, ${ }^{1}$ John Painter, ${ }^{1}$ Paul Douglas, ${ }^{2}$ Ibrahim Abubakar, ${ }^{3}$ Howard Njoo, \\ Chris Archibald, ${ }^{4}$ Jessica Halverson, ${ }^{4}$ John Robson, ${ }^{5}$ and Drew L. Posey ${ }^{1}$ \\ ${ }^{1}$ Division of Global Migration and Quarantine, National Center for Emerging and Zoonotic Infectious Diseases, \\ Centers for Disease Control and Prevention, Atlanta, GA, USA \\ ${ }^{2}$ Department of Immigration and Border Protection, Sydney, NSW, Australia \\ ${ }^{3}$ Public Health England and University College London, London, UK \\ ${ }^{4}$ Centre for Communicable Diseases and Infection Control, Public Health Agency of Canada, Ottawa, ON, Canada \\ ${ }^{5}$ Immigration New Zealand, Wellington, New Zealand
}

Correspondence should be addressed to Zachary White; zwhite@cdc.gov

Received 19 December 2016; Revised 22 February 2017; Accepted 16 March 2017; Published 23 March 2017

Academic Editor: Alexander S. Apt

Copyright (c) 2017 Zachary White et al. This is an open access article distributed under the Creative Commons Attribution License, which permits unrestricted use, distribution, and reproduction in any medium, provided the original work is properly cited.

Objective. Tuberculosis control in foreign-born populations is a major public health concern for Australia, Canada, New Zealand, United Kingdom, and the United States, large immigrant- and refugee-receiving countries that comprise the Immigration and Refugee Health Working Group (IRHWG). Identifying and comparing immigration and distribution of foreign-born tuberculosis cases are important for developing targeted and collaborative interventions. Methods. Data stratified by year and country of birth from 2005 to 2009 were received from these five countries. Immigration totals, tuberculosis case totals, and multidrug-resistant tuberculosis (MDR TB) case totals from source countries were analyzed and compared to reveal similarities and differences for each member of the group. Results. Between 2005 and 2009, there were a combined 31,785,002 arrivals, 77,905 tuberculosis cases, and 888 MDR TB cases notified at the federal level in the IRHWG countries. India, China, Vietnam, and the Philippines accounted for $41.4 \%$ of the total foreign-born tuberculosis cases and $42.7 \%$ of the foreign-born MDR tuberculosis cases to IRHWG. Interpretation. Collaborative efforts across a small number of countries have the potential to yield sizeable gains in tuberculosis control for these large immigrant- and refugee-receiving countries.

\section{Introduction}

Tuberculosis is one of the world's largest public health challenges. Approximately one-third of the world's population is infected with tuberculosis (TB), and an estimated 1.5 million people die from the disease each year [1]. The World Health Organization (WHO) established targets for TB control by 2035 [2]. These goals include targets for rates of diagnosis and treatment completion rates for persons inside a country [2]. However, migration is a factor that provides challenges for meeting targets as well as opportunities for control, particularly for countries with low incidence for $\mathrm{TB}$ [3].

Worldwide, there are approximately 232 million international migrants, or $3.2 \%$ of the world's population [4].
Although western, industrialized countries receive a large percentage of international migrants, other parts of the world also receive a large number of international migrants. Parts of the world in which $>10 \%$ of the population comprises international migrants include the Gulf countries, Eastern Europe, and even a few countries in Africa. Pertaining to TB, many of the top source countries for international migrants are also countries with a high burden of TB. For example, the top source country for international migrants in 2013 was India, a country with the highest burden of TB [1]. Other top countries for both international migrants and tuberculosis cases include the Russian Federation, China, Bangladesh, Pakistan, the Philippines, Afghanistan, and Indonesia.

Several of the world's largest immigrant- and refugeereceiving countries (Australia, Canada, New Zealand, 
the United States, and the United Kingdom) participate in the Immigration and Refugee Health Working Group (IRHWG), which aims to develop collaborative approaches for immigration and refugee resettlements, including TB. A majority of the TB cases in these countries are individuals who were born abroad, and the threat of multidrug-resistant (MDR) and extensively drug-resistant (XDR) TB is of significant concern [5-9]. Each participating country currently conducts TB screening overseas for immigrants (persons applying for permanent residency) and refugees; all members, except the United States, also perform TB screening overseas for persons who will live in the receiving country $>$ 6 months, also known as long-term visitors [10-14].

To address modern TB threats, each IRHWG country is in the process of improving TB prevention-control efforts, especially as it relates to immigrant and refugee populations with high burden of TB. To better identify the primary source countries that contribute the largest migrating populations and most TB cases to the group as a whole, we conducted an analysis of arrivals and TB case diagnoses.

\section{Methods}

We analyzed data on TB cases and arrivals to Australia, Canada, New Zealand, the United Kingdom, and the United States. The arrival data from each IRHWG country include long-term visitors, immigrant arrivals, and refugee arrivals; the arrival data were obtained through the immigration bureau for each country. The TB data are comprised of an in-country diagnosis of foreign-born TB cases; the TB case reporting came from the National Reporting Agencies in each IRHWG country. The authors acknowledge that the TB cases in this analysis are not directly linked to the immigration data presented.

All TB case data were stratified by year, birth country, and type of TB (treatment-sensitive or MDR). For analysis purposes, cumulative totals from 2005 to 2009 were utilized to determine the total volume of immigrants and diagnosed tuberculosis for each IRHWG country. Microsoft Excel and JMP 9 were used to construct tables in order to compare relationships among immigration totals, TB cases, and MDR TB cases. The top 20 source countries for total immigrants, TB cases, and MDR TB cases were determined using Microsoft Excel. Bar graphs were constructed using Microsoft Excel and $\mathrm{R}$ statistical software and tables made from Microsoft Excel and JMP.

Because a goal of this analysis is to develop a perspective as to which source countries are the largest ones for the IRHWG members as a whole, irrespective of the numbers of arrivals or cases, we calculated the average of the percentages for each source country. This was calculated for arrivals, foreign-born TB cases, and foreign-MDR TB cases. The calculations in the bar graphs display the burden (immigration volume, TB case volume, and MDR TB case volume) for each member country, as well as the burden to the group as a whole. For example, the average of each country's proportion of TB cases born in specific countries is shown in Figure 2 as a bar graph. Each country's proportion to the total TB burden is also represented in the graph.

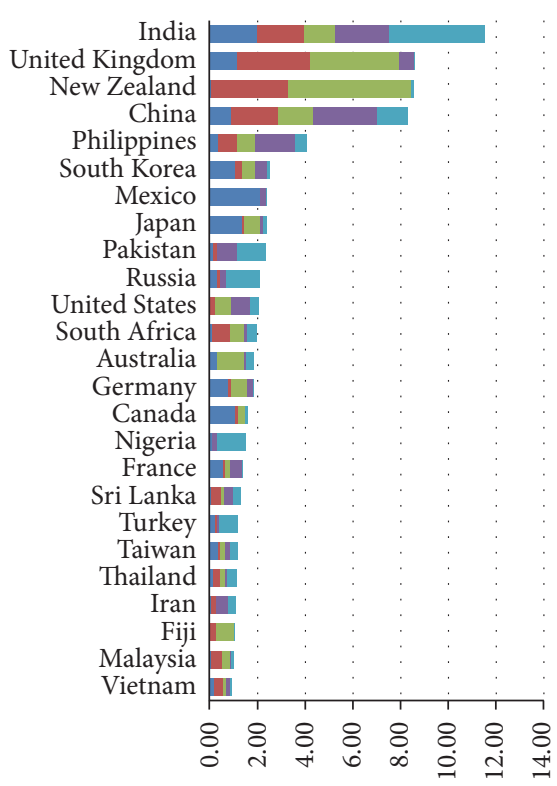

$(\%)$

- US proportion

- AUS proportion

- NZ proportion

- CAN proportion

- UK proportion

FIGURE 1: Combined averages of arrivals, 2005-2009.

Ethical Considerations. Ethical approval and informed consent were not required for this analysis.

\section{Results}

Between 2005 and 2009, there were 31,785,002 arrivals, 77,905 TB cases, and 888 MDR TB cases notified in these five countries. The data in Figures 1-3 represent the averages of each source country in respect to total arrivals, total TB cases, and total MDR TB cases from 2005 to 2009. The top five source countries for combined adjusted total arrivals (Figure 1) included India (11.5\%), United Kingdom (8.6\%), New Zealand (8.6\%), China (8.3\%), and the Philippines (4.1\%), while the top five source countries for combined adjusted total tuberculosis cases (Figure 2) were India (19.0\%), the Philippines (8.5\%), China (8.3\%), Vietnam (5.5\%), and Mexico (5.0\%). Similarly, the top five source countries for combined adjusted total MDR TB cases (Figure 3) were India (15.6\%), China (14.7\%), Papua New Guinea (8.5\%), the Philippines (6.8\%), and Vietnam (5.6\%).

Data from the analysis reveal that India, China, Vietnam, and the Philippines supplied the majority of diagnosed TB counts. These four countries accounted for combined adjusted $41.4 \%$ of the total foreign-born TB cases (Figure 2) and $42.7 \%$ of the foreign-born MDR tuberculosis cases (Figure 3). Of these, India was the leading source country for arrivals (11.5\% (Figure 1)), TB cases (19.0\% (Figure 2)), and MDR TB cases from 2005 to 2009 (15.6\% (Figure 3)).

Primary source countries for total immigrant arrivals, total TB cases, and total MDR TB cases varied for each of 


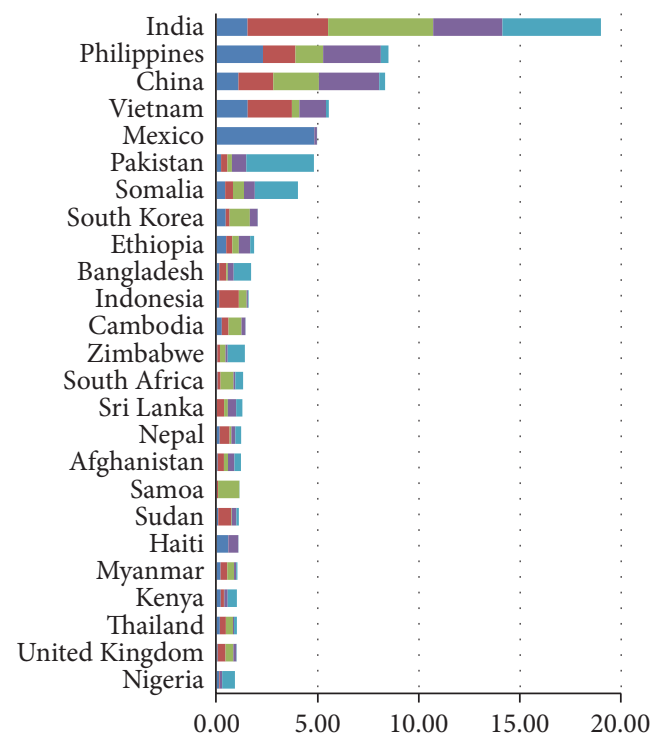

(\%)

- US proportion

- AUS proportion

- NZ proportion

- CAN proportion

- UK proportion

Figure 2: Combined averages of foreign-born TB cases, 2005-2009.

the five countries (Tables 1-3). Although multiple similarities were observed, the data reveal that four of the IRHWG countries have a specific source country/countries that contributes a significant amount of TB cases without affecting the group as a whole. Examples of specific source contributors include Papua New Guinea (3.4\% of foreign-born TB cases and $42.4 \%$ of MDR TB cases) for Australia; Samoa (5.3\% TB cases) for New Zealand; Somalia (10.6\% TB cases and 12.3\% MDR TB cases) and Pakistan (16.7\% TB cases, $8.7 \%$ MDR TB cases) for the United Kingdom; and Mexico (24.1\% TB cases and 13.7\% MDR TB cases) for the United States (Tables 1, 2, and 3). Canada received the majority of cases from the four largest source countries (India, the Philippines, China, and Vietnam) but did not have a unique source country that was uncommon to the other members. In this analysis, instead of grouping countries by regions, we examined data for the individual source country to understand their specific impact on our immigration and TB programs.

\section{Discussion}

In this analysis, India, China, Vietnam, and the Philippines contributed the largest proportion of foreign-born TB cases to Australia, Canada, New Zealand, United Kingdom, and the United States as a whole during 2005-2009. The data used in this analysis is a couple of years old, but the immigration and TB trends are similar to the results of this analysis. The four countries accounted for $41.4 \%$ of combined adjusted foreignborn $\mathrm{TB}$ cases and $42.7 \%$ of the combined adjusted foreignborn MDR TB cases during this period of time. Each of these

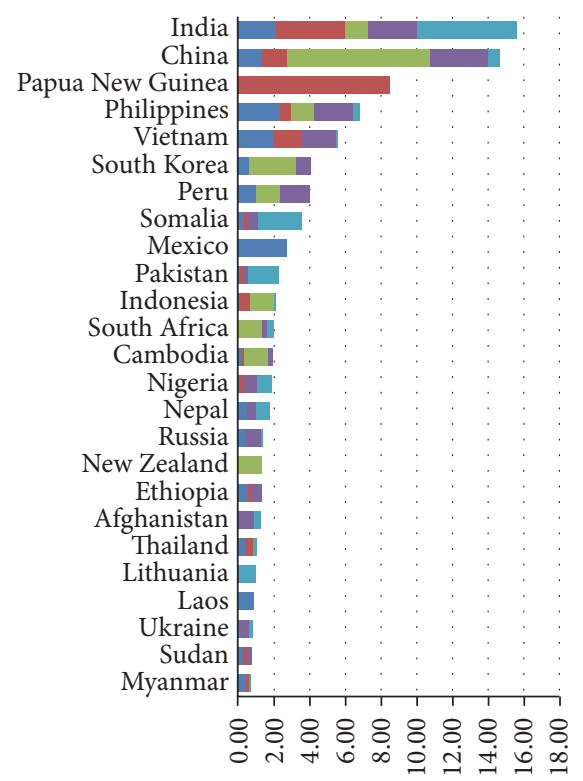

(\%)

- US proportion

- AUS proportion

- NZ proportion

- CAN proportion

- UK proportion

FIGURE 3: Combined averages of MDR TB cases, 2005-2009.

four countries is also included in the 2015 WHO list of highburden countries with respect to TB [1].

However, these data also highlight the unique source countries for each of these countries, which illustrate differences in migration patterns. These unique situations may reflect factors related to geography, such as Mexico as a source country for the United States, or issues related to longstanding historical ties, such as Pakistan for the United Kingdom.

Although each country publishes immigration and $\mathrm{TB}$ surveillance figures, and the importance of foreign-born TB cases in industrialized countries has been described [15], this is the first comparison of immigration and TB data among large immigrant- and refugee-receiving countries to our knowledge.

The results of this analysis also mirror global comparisons over time. Just as the global trends in international migration have increased over time, so the volumes of arrivals have increased too during the study period. The growing importance of Asian arrivals, in particular, is also reflected in this analysis. In the United States, for example, the highest proportion of immigrants to the United States transitioned from Hispanics to Asians in 2009 [16].

The results also reflect global trends in $\mathrm{TB}$, as many of the top birth countries for TB cases are also WHO high-burden countries. Although only five receiving countries are included in this analysis, the similarities (Figures 1-3) underscore the need to develop collaborative strategies to address the burden of TB in migrating populations [17]. 


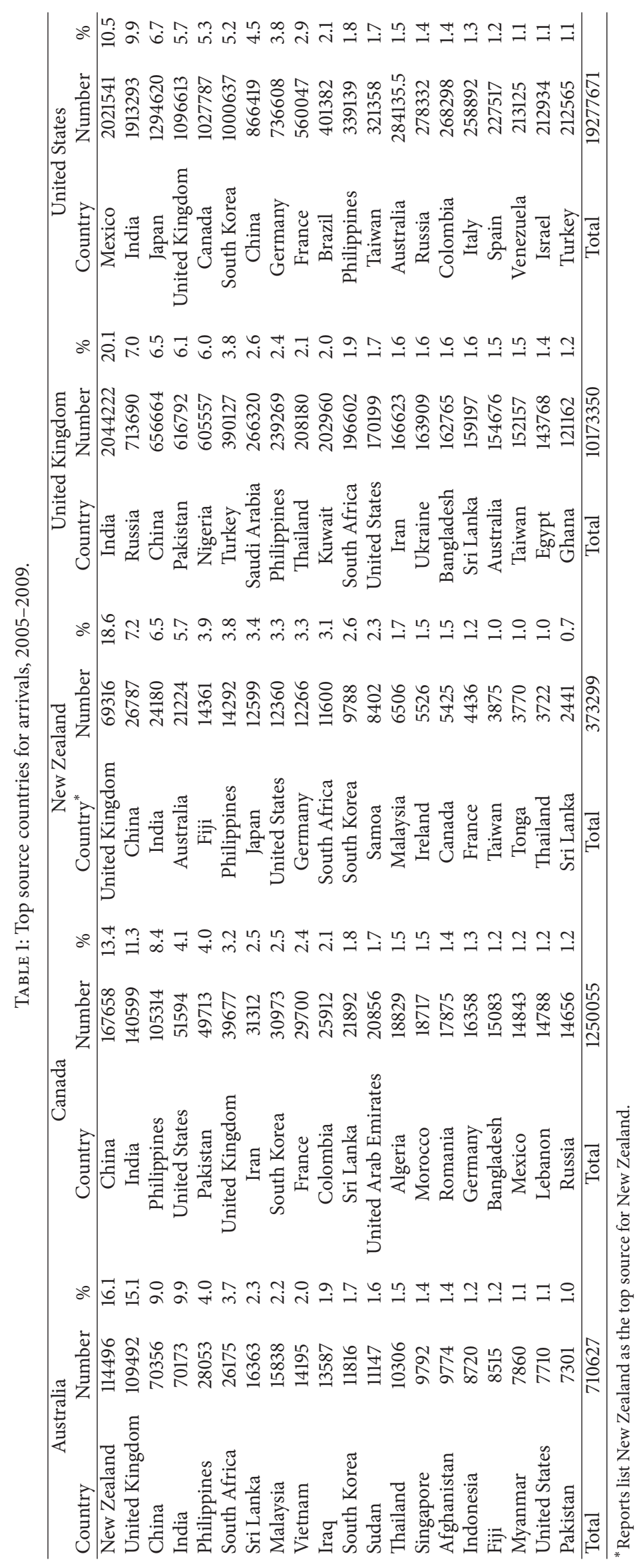




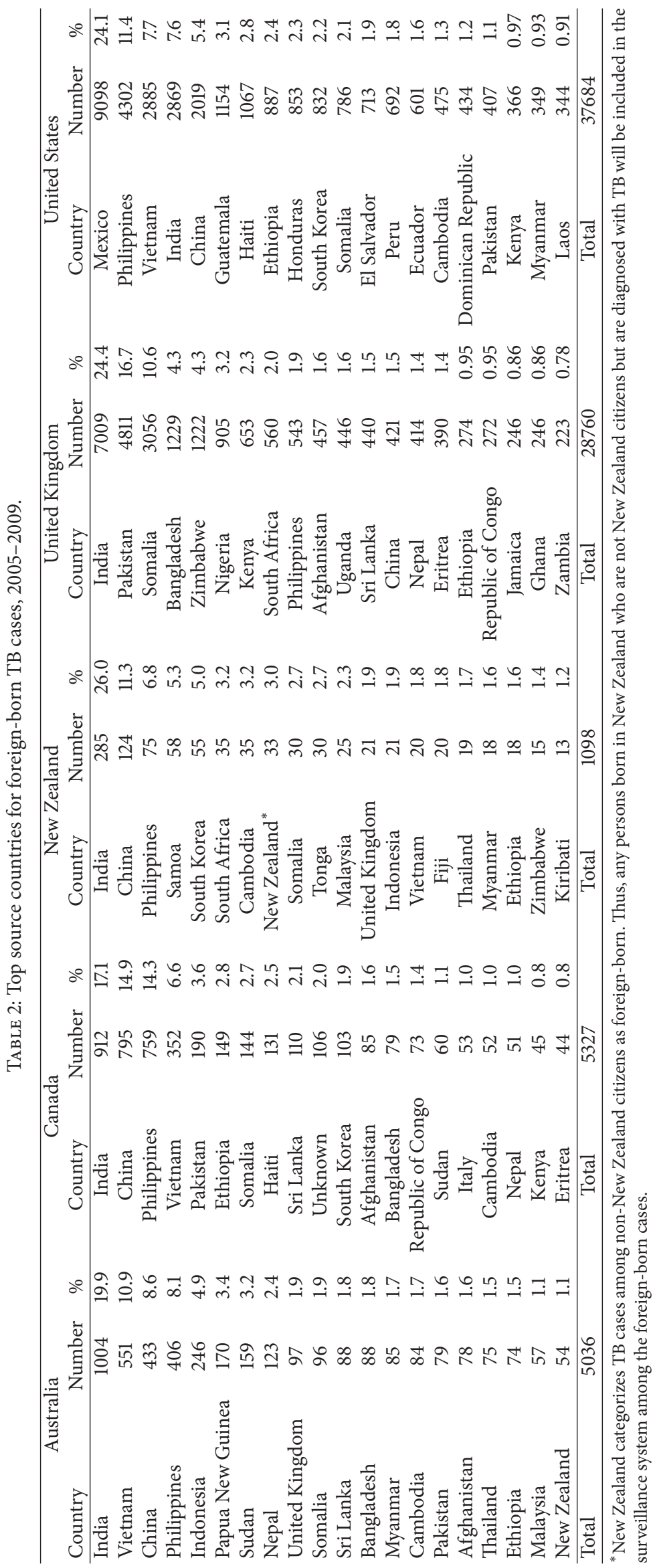




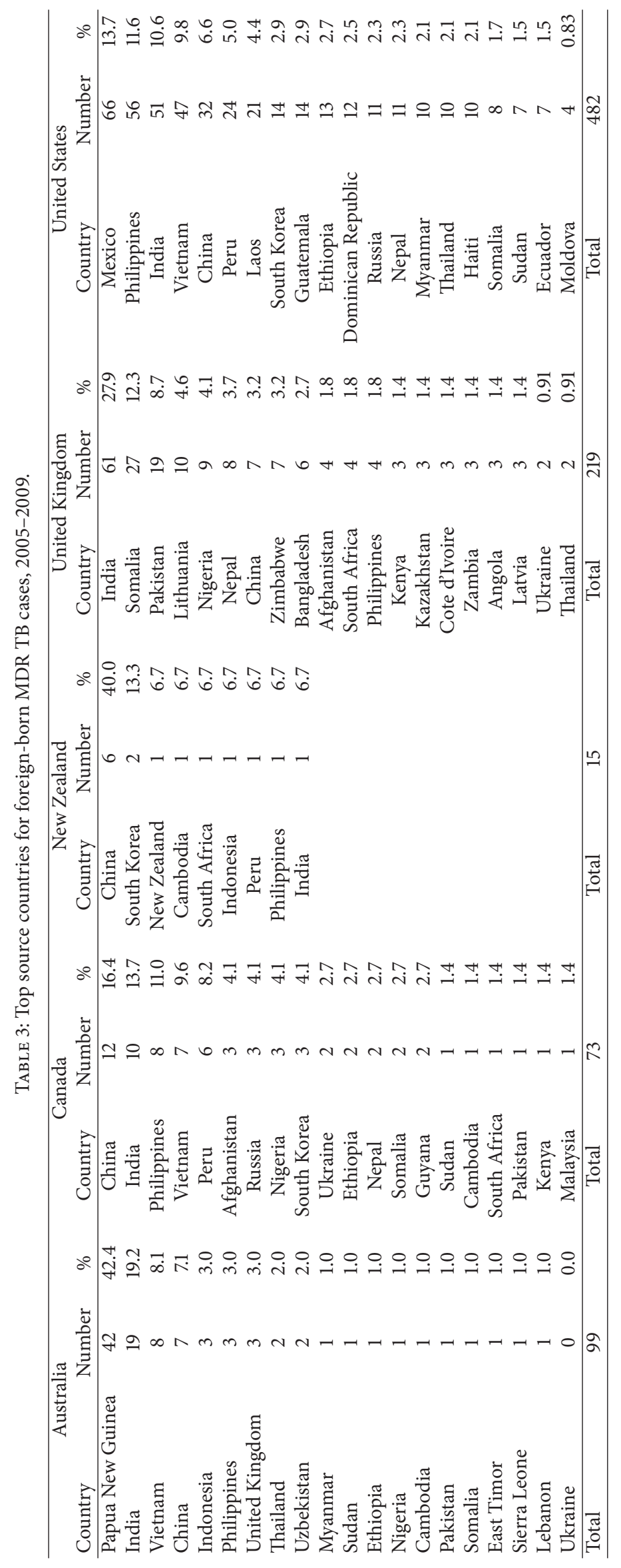


During the period of this analysis, an average of 6 million persons were admitted annually to the five participating countries. Currently, approximately 2 million applicants for migration status to these five countries are screened for TB annually; the foreign-born TB cases which occur may or may not be discovered through the screening process. These examinations are performed by $>2,000$ panel physicians worldwide. Panel physicians are medical doctors who have agreements with each country to conduct the migrant medical exam overseas. Each of the IRHWG countries require overseas TB screening for all immigrants and refugees and each except for the United States routinely requires screening for long-term visitors. Moreover, all but the United Kingdom have historically required this screening [18]; following a successful pilot program in a limited number of countries, the United Kingdom began a targeted overseas screening program in 2012 for applicants from high-incidence countries [19]. Screening applicants for TB is very effective at preventing importation of active $\mathrm{TB}$ cases into the receiving countries $[20,21]$.

Managing a modern overseas TB screening program for migration and refugee resettlements results in development of laboratory and treatment capacity. The TB screening algorithms of IRHWG countries are similar in that they either require (United States) or use (Australia, Canada, New Zealand, and United Kingdom), where available, TB cultures for those suspected of having active pulmonary TB, drug susceptibility testing (DST) on positive isolates, and treatment delivered as directly observed therapy (DOT) prior to entry [10-14]. Since 2007, implementation of the US program's culture and DOT requirement has resulted in additional culture and DST laboratories as well as increased training for personnel involved with TB control [21, 22].

While each of these country's efforts in managing TB screening programs is invaluable for reducing importation of TB, they should also be leveraged to assist with control efforts within source countries. TB elimination in receiving countries is difficult without addressing TB in foreignborn populations [23]. For this reason, it is hoped that improved linkages between panel physician activities and TB control efforts within their countries would benefit the migrants and others in their source populations. This analysis helps demonstrate the fact that because panel physician volumes are large in key source countries, these countries are uniquely positioned to have their investments in the screening program also contribute to local control efforts [24]. And this analysis helps determine for which countries that contribution could be most needed. Additional benefits to screening programs could possibly be achieved by having panel physicians develop relationships with their TB controllers, share information on their experiences, share laboratory capacity, and comanage TB cases where DOT capacity is scarce.

The authors expected India to be a common source country but were surprised that the average number of cases was more than twice as high as that for the next two countries, the Philippines and China. The participating countries have collaborated closely with India, and this country was the first country for implementation of the United Kingdom screening program. However, the results of this analysis highlight the importance of collaborations with India for migrants, as well as for helping with overall TB control in India, since it is the highest-burden country for TB worldwide.

In many instances, $\mathrm{TB}$ treatment for those who travel to the US may not occur until years after they have arrived. While the data in this analysis shares source country of the migrants, it does not automatically imply importation. Individuals may acquire TB infection during travel or in the United States upon arrival.

Although this analysis yields important findings regarding migration and TB, there are some limitations. First, data for this analysis were only requested from 2005 to 2009, which is a short period of time. If additional years were observed for the analysis, additional trends for each country and the group as a whole might have been exposed. However, the data from these five countries for subsequent years suggest similar burdens of arrivals and diagnoses. Second, the analysis only included data for five countries. While these are some of the largest immigrant-receiving countries, additional future analyses should include countries outside the group. The analysis could have used data to separate the arrivals and TB cases by visa types for each receiving country. This would allow stratification of the arrivals and cases by populations to see which had the highest $\mathrm{TB}$ rates within each country. It is also important to note that the TB data were not stratified by time since arrival. Thus, the potential exists that trends among this subset may be slightly different. Finally, while this analysis can be used to help participating countries in the management of their screening programs by indicating which resources could be targeted, a cost-effectiveness review of screening programs on potential collaborations was beyond the scope of this analysis.

Australia, Canada, New Zealand, the United Kingdom, and the United States are the largest immigrant- and refugeereceiving countries in the world and are currently collaborating on preventing importation of TB into each of their countries. Joint efforts in a small number of high-burden countries can help prevent importation of TB cases and also contribute to control efforts within source countries.

\section{Disclosure}

The findings and conclusions of this article are those of the authors and do not necessarily represent the official position of the Centers for Disease Control and Prevention.

\section{Conflicts of Interest}

The authors declare no conflicts of interest regarding the publication of this paper.

\section{Authors' Contributions}

Zachary White, John Painter, and Drew L. Posey were involved in the conception and design and performed the analysis. Paul Douglas, Ibrahim Abubakar, Howard Njoo, Chris Archibald, Jessica Halverson, and John Robson were 
involved in the study implementation, interpreted the data, and provided important intellectual input. Zachary White and Drew L. Posey wrote the first draft.

\section{Acknowledgments}

The authors thank Mr. Terry Comans for assistance garnering support for the analysis among participating countries; Mr. David Watt for assistance with data from Australia; Dr. Tom Navin for US TB case data; Mr. Yecai Liu for identifying long-term visitors in US arrival data; the panel physicians for performing overseas TB screening; and Ms. Courtney Chappelle for mapping activities. The authors acknowledge the support from Centers for Disease Control and Prevention, Public Health England, Department of Immigration and Border Protection Australia, Public Health Agency of Canada, and Immigration New Zealand.

\section{References}

[1] World Health Organization, Global Tuberculosis Report 2015, WHO, Geneva, Switzerland, 2015, http://www.who.int/tb/ publications/global_report/en/.

[2] Global Strategy and Targets for Tuberculosis Prevention, Care and Control After 2015, May 2014, http://www.who.int/tb/ post2015_strategy/en/.

[3] K. Lonnroith, G. B. Migliori, I. Abubakar et al., "Towards tuberculosis elimination: an action framework for low-incidence countries," European Respiratory Journal, vol. 45, no. 4, pp. 928952, 2015

[4] Department of Economic Affairs Population Division, International Migration Report 2013, United Nations Doc ST/ESA/ SER.A/346, 2013.

[5] CDC, Reported Tuberculosis in the United States, 2012, U.S. Department of Health and Human Services, CDC, Atlanta, Ga, USA, 2013.

[6] C. Barry, J. Waring, R. Stapledon et al., "Tuberculosis notifications in Australia, 2008 and 2009," Communicable Disease Intelligence, vol. 36, no. 1, pp. 82-94, 2012, http://www.health .gov.au/internet/main/publishing.nsf/Content/cda-cdi3601c.htm\# refs.

[7] Tuberculosis Prevention and Control in Canada, Centre for Communicable Disease and Infection Control, Public Health Agency of Canada, 2014, http://www.phac-aspc.gc.ca/.

[8] E. Lim and H. Heffernan, Tuberculosis in New Zealand: Annual Report 2012, Institute of Environmental Science and Research (ESR), Porirua, New Zealand, 2013.

[9] Tuberculosis in the UK: Annual Report on Tuberculosis Surveillance in the UK, Public Health England, London, UK, 2013.

[10] Department of Immigration and Citizenship, Global Health, Sydney, Australia, Instructions for medical and radiological examination of Australian visa applicants, Australian Government, March 2012.

[11] Government of Canada, Citizenship and Immigration Canada: Immigration and Medical Examination Instructions, 2013, http://www.cic.gc.ca/english/department/partner/pp/pdf/IMEI_ Tuberculosis.pdf.

[12] Ministry of Health, Guidelines for Tuberculosis Control in New Zealand 2010, Ministry of Health, Wellington, New Zealand, 2010, http://www.moh.govt.nz.
[13] UK Visas \& Immigration: UK Tuberculosis Technical Instructions (UKTBTI), Public Health England, September 2013 Version 6, https://www.gov.uk/government/uploads/system/ uploads/attachment_data/file/245009/UK_tuberculosis_technical_instructions.pdf.

[14] CDC, CDC Immigration Requirements: Technical Instructions for Tuberculosis Screening and Treatment, U.S. Department of Health and Human Services, Atlanta, Ga, USA, 2007, http:// www.cdc.gov/immigrantrefugeehealth/pdf/tuberculosis-ti-2009 .pdf.

[15] K. P. Cain, S. R. Benoit, C. A. Winston, and W. R. Mac Kenzie, "Tuberculosis among foreign-born persons in the United States," The Journal of the American Medical Association, vol. 300, no. 4, pp. 405-412, 2008.

[16] Pew Research Center, The Rise of Asian Americans, 2013, http://www.pewsocialtrends.org/files/2013/04/Asian-Americansnew-full-report-04-2013.pdf.

[17] B. K. Moore, D. L. Posey, S. A. Maloney, M. Cetron, and K. Castro, Tackling Tuberculosis Abroad-The Key to TB Elimination in the United States. June 2014. A Report of the CSIS Global Health Policy Center, Center for Strategic and International Studies, 2014, https://csis.org/files/publication/140604_Moore_ TacklingTBAbroad_Web.pdf.

[18] M. Dara, B. D. Gushulak, D. L. Posey, J.-P. Zellweger, and G. B. Migliori, "The history and evolution of immigration medical screening for tuberculosis," Expert Review of AntiInfective Therapy, vol. 11, no. 2, pp. 137-146, 2013.

[19] UK Border Agency, "New screening to tackle tuberculosis in the UK," http://www.ukba.homeoffice.gov.uk/sitecontent/ newsarticles/2012/may/42-tb-test.

[20] Y. Liu, M. S. Weinberg, L. S. Ortega, J. A. Painter, and S. A. Maloney, "Overseas screening for tuberculosis in U.S.-bound immigrants and refugees," New England Journal of Medicine, vol. 360 , no. 23, pp. 2406-2415, 2009.

[21] D. L. Posey, M. P. Naughton, E. A. Willacy et al., "Implementation of new TB screening requirements for U.S.-bound immigrants and refugees-2007-2014," Morbidity and Mortality Weekly Report, vol. 63, pp. 234-236, 2014.

[22] Y. Liu, D. L. Posey, M. S. Cetron, and J. A. Painter, "Effect of a culture-based screening algorithm on tuberculosis incidence in immigrants and refugees bound for the United States a population-based cross-sectional study," Annals of Internal Medicine, vol. 162, no. 6, pp. 420-428, 2015.

[23] A. N. Hill, J. E. Becerra, and K. G. Castro, "Modelling tuberculosis trends in the USA," Epidemiology and Infection, vol. 140, no. 10, pp. 1862-1872, 2012.

[24] K. Schwartzman, O. Oxlade, R. Graham Barr et al., "Domestic returns from investment in the control of tuberculosis in other countries," The New England Journal of Medicine, vol. 353, no. 10, pp. 1008-1020, 2005. 


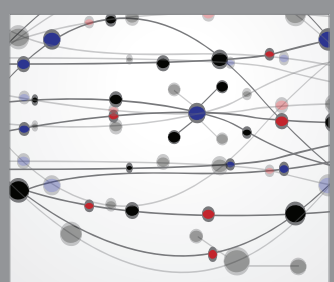

The Scientific World Journal
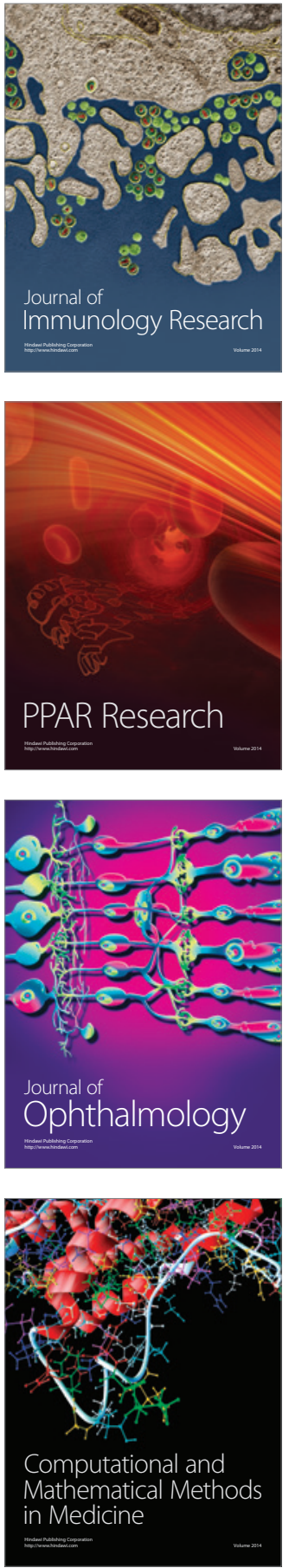

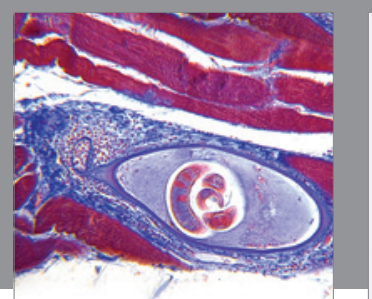

Gastroenterology Research and Practice
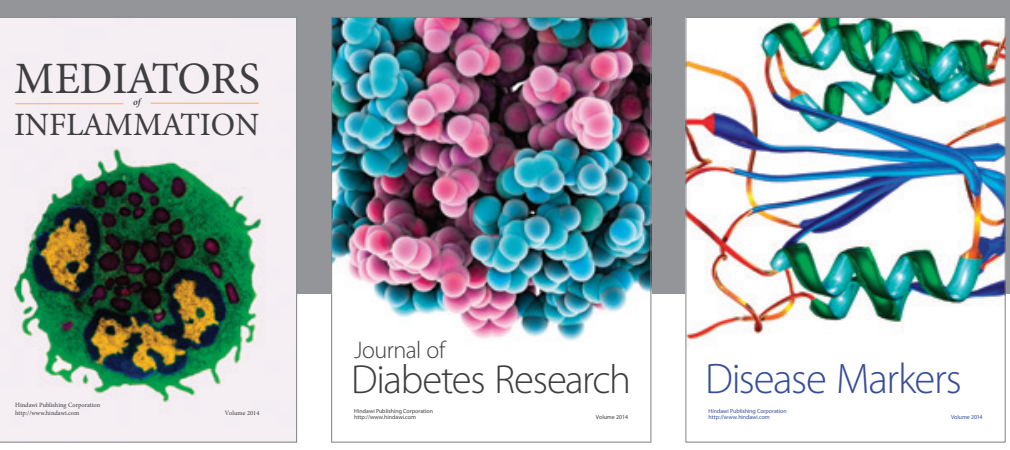

Disease Markers

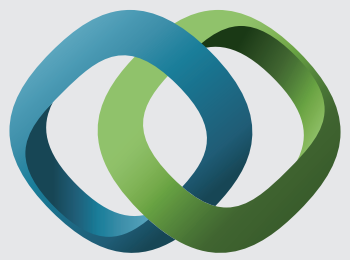

\section{Hindawi}

Submit your manuscripts at

https://www.hindawi.com
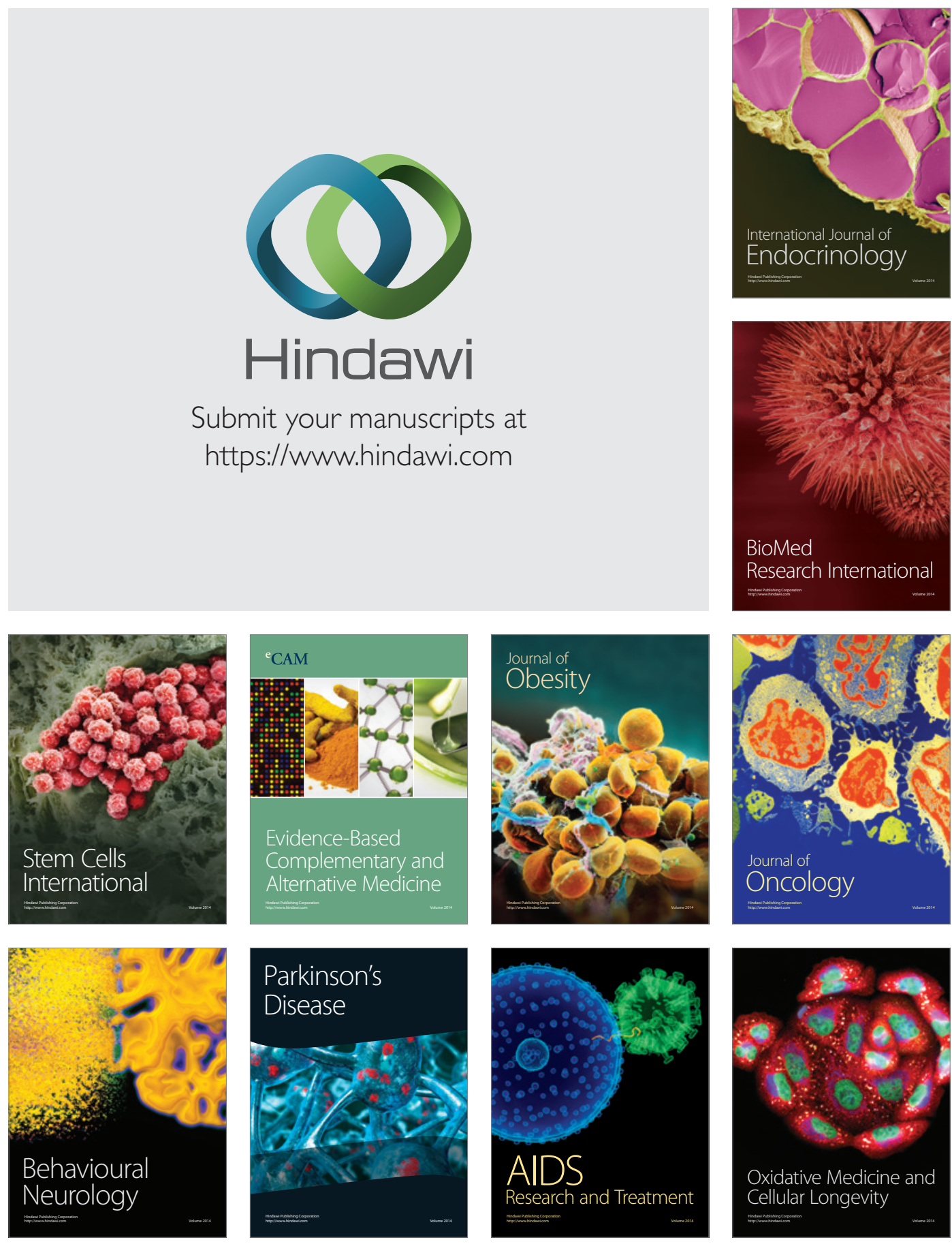